\title{
Swimmer simulation using robot manipulator dynamics under steady water
}

\author{
Kazunori Shinohara \\ Kanagawa Academy of Science and Technology, Kawasaki City, Kanagawa, Japan; shinohara@06.alumni.u-tokyo.ac.jp
}

Received 1 July 2010; revised 5 August 2010; accepted 13 August 2010.

\section{ABSTRACT}

To help swimmers improve, we have developed a computational swimming model using underwater manipulator dynamics. We formulate the equations of the underwater manipulator dynamics using the fluid drag, which is proportional to the square of the velocity. We construct a swimming model consisting of several links based on these equations. The distance traveled by the optimal swimming motion is derived using the model. The input parameters are the joint torques. The arm and leg positions in the model are determined from the joint torques. The force transmitted from the water to the manipulator is defined to be the action force, and the force transmitted from the manipulator to the water is defined to be the reaction force. This reaction force is defined to be the propulsion force. By combining the propulsion force generated by the arms and legs and the frictional drag with respect to the body we can calculate the distance traveled. To optimize the propulsion, which depends on the swimmer's motion, a variational approach using the Lagrange function is applied. We can use the model to simulate 2D pseudo-backstroke motion. Our model has a lower cost than other techniques in the literature, because it does not require computational fluid dynamics (CFD). The swimmer velocity calculated by our model agrees quite closely with the results in the literature. The model qualitatively captures the movement of an actual swimmer.

Keywords: Sports Engineering; Swimmer; Robot Manipulator Dynamics; Optimal Trajectory; Adjoint Variable Method; Euler-Lagrange Equation; Fluid Drag Force; Variational Method

\section{INTRODUCTION}

In highly specialized sports such as Olympic-level swimming, different competitors have similar skill levels. Therefore, studies that develop new concepts are important for producing new records. These studies mainly focus on the physics, physiology, and psychology [1-7]. Our study instead focuses on engineering; the goal of engineering studies is to develop software and hardware tools that provide a competitive advantage.

Swimmer simulation has mainly focused on applications in the amusement industry such as movies and video games. Low-cost algorithms have been developed that carry out, for example, motion capture from an animated image. Using such techniques, the realism of the visualization has been greatly advanced [8]. These techniques are not appropriate outside the amusement industry because the dynamics are largely ignored.

To help swimmers improve, the dynamics must reveal the relationship between the propulsion and the swimmer's motion. The swimmer receives water pressure on the body, and the pressure distribution on the surface of the body has been obtained by CFD simulation techniques. A simulation model called SWUM has been developed; this model consists of the entire human body and was developed under unsteady flow [9]. The CFD

In highly specialized sports such as Olympic game, there is little difference in skill level.

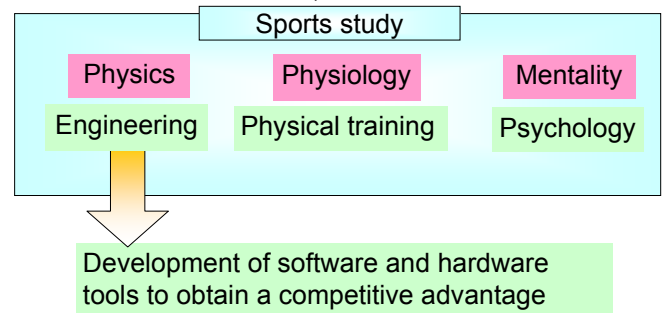

Figure 1. Sports engineering. 
Fluent software has been used to analyze the effect of the position of a swimmer's head within a Reynolds number under turbulence [10]. The propulsive contribution of the swimmer's upper arm and the effect generated by body roll movement have been revealed [11]. During submerged gliding in swimming, the effect of the body position on the drag coefficient has been analyzed using a computational fluid dynamics methodology $[12,13]$. Initial simulations of towing using SPH for both male and female swimmers have been presented [14]. The effect of the hand on the propulsion force during swimming has been investigated using CFD software [15-17].

The aim of this study is to develop a swimming simulator based on the dynamics. In the first step of the simulation, a test model using one leg of the swimmer is constructed to calculate the propulsion. A swimming model is then built based on the test model to compute the human motion [18]. Finally, by considering the friction of the swimmer's body in the model [19], we find that the results qualitatively agree with actual measurements.

\section{SWIMMER REPRESENTATION AND ASSUMPTIONS}

The swimmer representation is shown in Figure 2; it is a minimalist representation.

The model is bilaterally symmetric and has ten links.

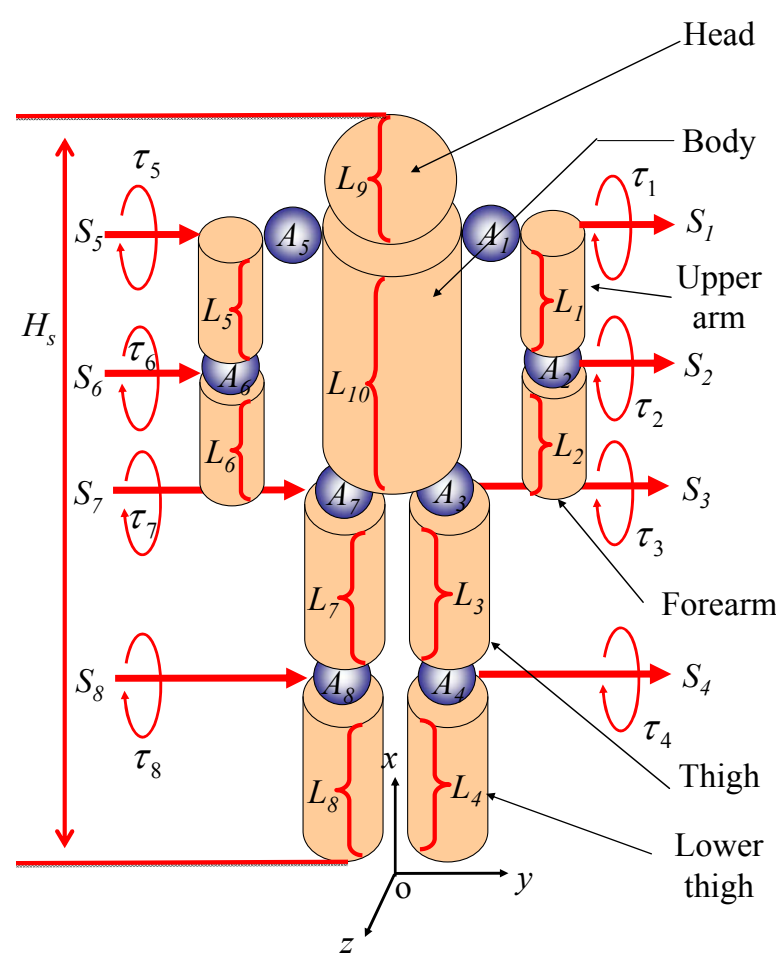

Figure 2. Swimmer representation.
The link numbers $l_{9}$ and $l_{10}$ represent the head and body. On the right side, the link numbers $l_{1}, l_{2}, l_{3}$, and $l_{4}$ represent the upper arm, forearm, thigh, and lower thigh respectively. Joints $A_{1}, A_{2}, A_{3}$, and $A_{4}$ represent the joints between the body and upper arm, between the upper arm and forearm, between the body and thigh, and between the thigh and lower thigh, respectively. The link structure on the left side is similar to that on the right. The torque of joint $A_{i}(i=1, \ldots, 8)$ is defined to be $\tau_{i}(i=1, \ldots, 8)$.

The other main variables are defined as follows:

$\theta_{i}$ : Angle at the tip of link $i$ at joint $A_{i}$;

$\omega_{i}$ : Angular velocity vector at the tip of link $i$ at joint $A_{i}$. This vector represents $\omega_{i}=\left(0, \dot{\theta}_{i}, 0\right)$ in assumption 2 below;

$\boldsymbol{l}_{i}$ : Longitudinal position vector from joint $A_{i}$ to the tip of link $l_{i}$;

$s_{i}$ : Rotation axis unit vector of joint $A_{i}$;

${ }^{G} \boldsymbol{l}_{\boldsymbol{i}}$ : Longitudinal position vector from the origin of $A_{i}$ to the barycenter in link $l_{i}$;

${ }^{\boldsymbol{G}} \boldsymbol{v}_{\boldsymbol{i}}$ :Translational velocity vector of $\operatorname{link} l_{i}$;

${ }^{G} \dot{\boldsymbol{v}}_{\boldsymbol{i}}$ : Translational acceleration vector of link $l_{i}$;

$D_{i}$ : Diameter of link $l_{i}$;

$C_{i}$ : Drag coefficient of link $l_{i}$;

$m_{i}$ : Mass of link $l_{i}$;

$\rho$ : Density of fluid;

$V_{i}$ : Volume of link $l_{i}$;

$L_{i}$ : Length of link $l_{i}$

In this paper, the assumptions are as follows:

Assumption 1: Links are defined to be rigid bodies.

Assumption 2: The motions of the arms and legs are assumed to be 2D in the $\mathrm{x}-\mathrm{z}$ plane. Motion does not occur in the $y$ direction in Figure 2.

Assumption 3: The fluid drag with respect to the longitudinal direction of the links is assumed to be negligible.

Assumption 4: The fluid around the swimmer is assumed to be stationary and steady.

Assumption 5: The head is defined to be a sphere. The other body parts are defined to be circular cylinders. Parameters $D_{i}$ and $L_{i}$, represent the diameter and length, respectively, of cylinder $i$.

Assumption 6: The body moves forward in the $x$ direction in Figure 2.

Assumption 7: The velocity and acceleration of the body do not affect the torque $\tau_{i}$ of joint $A_{i}$. The velocity vector $\boldsymbol{v}_{0}$ at the body is assumed to be zero, except in Eq.9.

Assumption 8: The link structures $\left(l_{1}, l_{2}\right),\left(l_{3}, l_{4}\right),\left(l_{5}, l_{6}\right)$, and $\left(l_{7}, l_{8}\right)$ are assumed to be independent of each other.

\section{SWIMMER DYNAMICS EQUATIONS}

\subsection{Fluid Drag}

When a swimmer swims in water, the swimmer receives external forces from the fluid. By integrating with resp- 
ect to the longitudinal length of link $l_{i}$ we can determine these forces as follows:

$$
\boldsymbol{d}_{\boldsymbol{i}}=\frac{\rho}{2} C_{i} D_{i} \int_{0}^{L_{i}}\left|\boldsymbol{v}_{i-1}+\boldsymbol{\omega}_{\boldsymbol{i}} \times{ }^{G} \boldsymbol{l}_{\boldsymbol{i}}\right|\left(\boldsymbol{v}_{i-1}+\boldsymbol{\omega}_{i} \times{ }^{G} \boldsymbol{l}_{i}\right) \boldsymbol{d} \boldsymbol{l}_{\boldsymbol{i}}
$$

where the drag coefficients $C_{i}$ depend on the link shape and are defined as functions of the Reynolds number.

\subsection{Underwater Manipulator Dynamic Equation}

The underwater manipulator dynamic equation of two links is formulated as described in assumption 8 . The subscript in Eqs. 2 to 5 represents the relationship between links $l_{1}(i=1)$ and $l_{2}(i=2)$, between links $l_{5}(i=1)$ and $l_{6}(i=2)$, between links $l_{3}(i=1)$ and $l_{4}(i=2)$, and between links $l_{7}(i=1)$ and $l_{8}(i=2)$, respectively. The force ${ }^{G} \boldsymbol{f}_{i}$ with respect to the barycenter $G$ in the link is:

$$
{ }^{G} \boldsymbol{f}_{i}=m_{i}\left({ }^{G} \boldsymbol{\alpha}_{i}-\boldsymbol{g}\right)+\rho V_{i} \boldsymbol{g}
$$

where ${ }^{G} \boldsymbol{\alpha}_{i}$ represents the acceleration with respect to the barycenter $G$. The vectors $\boldsymbol{g}$ and $\rho V_{i} \boldsymbol{g}$ for link $i$ represent the gravity and buoyancy, respectively. The force at the tip of link $i$ is the resultant force consisting of the force generated from link $i+1$ to link $i$, the force of Eq.3, and the drag of Eq.1:

$$
\boldsymbol{f}_{i}=\boldsymbol{f}_{i+1}+{ }^{G} \boldsymbol{f}_{\mathrm{i}}+\boldsymbol{d}_{i}
$$

Using Eqs.2 and 4, we define the moment at the tip of link $i$ as follows:

$$
\boldsymbol{n}_{\boldsymbol{i}}=\boldsymbol{n}_{i+1}+\boldsymbol{l}_{i} \times \boldsymbol{f}_{i+1}+{ }^{G} \boldsymbol{l}_{\boldsymbol{i}} \times{ }^{G} \boldsymbol{f}_{i}+{ }^{G} \boldsymbol{n}_{i}
$$

where the variable ${ }^{G} \boldsymbol{l}_{\boldsymbol{i}}$ represents the position vector with respect to the barycenter. The torques with respect to the rotation axis direction are as follows:

$$
\tau_{i}=\boldsymbol{s}_{i}^{T} \boldsymbol{n}_{i}
$$

where the superscript $T$ represents transposition.

\subsection{Propulsion Force}

The mechanism of the propulsion force is shown in Figure 3. The link is assumed to be fixed to the ground through the joint. The rotation of a link causes fluid drag on the manipulation surface. The force acts from the fluid to the structure. If the link is not fixed to the ground, the force acts from the fluid to the structure and at the same time reacts from the structure to the fluid. The link moves as a result of the reaction force. This reaction force is defined to be the propulsion force, and the body receives friction underwater. Therefore, the propulsion force is defined to be

$$
P=R-E
$$

where $R$ represents the reaction force and $E$ represents the friction.

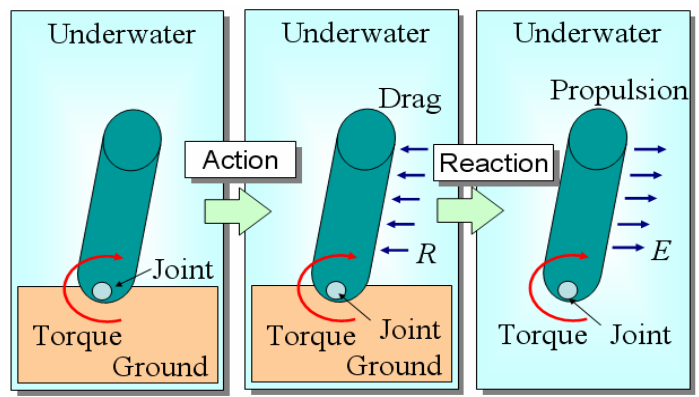

Figure 3. Mechanism of propulsion force.

\subsection{Reaction Force}

Using Eq.1, the reaction force at link $l_{i}(i=1, \ldots, 8)$ is defined as follows:

$$
\sum_{i=1}^{8} \boldsymbol{d}_{\boldsymbol{i}}=\sum_{i=1}^{8} \frac{\rho}{2} C_{i} D_{i} \int_{0}^{L_{i}}\left|\boldsymbol{v}_{\boldsymbol{i - 1}}+\boldsymbol{\omega}_{\boldsymbol{i}} \times{ }^{G} \boldsymbol{l}_{\boldsymbol{i}}\right|\left(\boldsymbol{v}_{\boldsymbol{i}-\mathbf{1}}+\boldsymbol{\omega}_{\boldsymbol{i}} \times{ }^{G} \boldsymbol{l}_{\boldsymbol{i}}\right) \boldsymbol{d} \boldsymbol{l}_{\boldsymbol{i}}
$$

As described in assumption 2, vector $\boldsymbol{d}_{\mathrm{i}}$ has $y$ and $z$ components that are zero. Therefore, this vector is replaced by a scalar. The reaction force $R$ of the model is estimated with respect to the $x$ direction:

$$
R=\sum_{i=1}^{8} d_{i}
$$

\subsection{Friction}

The velocity of a swimmer depends on the drag and propulsion [20]. Takagi et al. developed a device that can measure the drag in swimming [19]. They try to quantify the drag acting on a self-propelling swimmer. This active drag $D_{a}$ is assumed to consist of passive drag and kinetic drag. The active drag $D_{a}$ is estimated using experimental results as follows:

$$
\begin{aligned}
& \boldsymbol{D}_{a}=0.015 \rho A_{s} \boldsymbol{v}_{0}^{2} \\
& +\frac{1}{2} \rho V_{s}^{\frac{3}{2}} \boldsymbol{v}_{0}^{2}\left\{0.86 \exp \left(-3.9 \frac{\left|\boldsymbol{v}_{0}\right|}{\sqrt{g H_{s}}}\right)\right\}
\end{aligned}
$$

where $A_{s}, V_{s}$, and $H_{s}$ represent the surface, volume, and height of the swimmer, respectively. Vector $\boldsymbol{v}_{0}$ represents the velocity of the body $\left(l_{10}\right)$. As described in assumption 6 , vector $\boldsymbol{v}_{0}$ has $y$ and $z$ components that are zero. The friction in the $x$ direction is equal to $E$ in Eq.6 as follows:

$$
E=D_{a}
$$

Using Eqs.8 and 10, we can calculate the propulsion force of the swimmer via Eq.6. The calculation procedures are summarized in Figure 4.

\subsection{Evaluation of Distance Traveled}

The swimmer acceleration $\dot{v}_{0}$ can be calculated as fol- 
lows:

$$
\dot{v}_{0}=\frac{P}{M}=\frac{1}{M}(R-E)
$$

where $M$ represents the mass and $P$ represents the propulsion of the swimmer. By integrating Eq.12 with respect to time, we can calculate the velocity and distance as follows:

$$
\begin{aligned}
v_{0} & =\int_{0}^{t} \dot{v}_{0} d t \\
x & =\int_{0}^{t} v_{0} d t
\end{aligned}
$$

where $x$ represents the distance traveled by the swimmer, as shown in Figure 2. acceleration and velocity of the initial condition $(t=0.0 \mathrm{~s})$ are defined to be zero.

\section{ALGORITHM}

\subsection{Purpose}

In this study, the optimal trajectory of the manipulator is calculated. The process from the input of the torque to the output of the distance traveled is as follows:

Process 1: Parameters and initial conditions are set in the model.

Process 2: The time history of the torques from start time 0 to end time $t$ is set in the joints.

Process 3: Angular accelerations are calculated at each joint.

Process 4: Angular velocities are calculated at each joint.

Process 5: Angles are calculated at each joint.

Process 6: The propulsion forces $R$ of the arms and legs are calculated using Eq.8.

Process 7: The acceleration $\dot{v}_{0}$ is calculated using Eq.11.

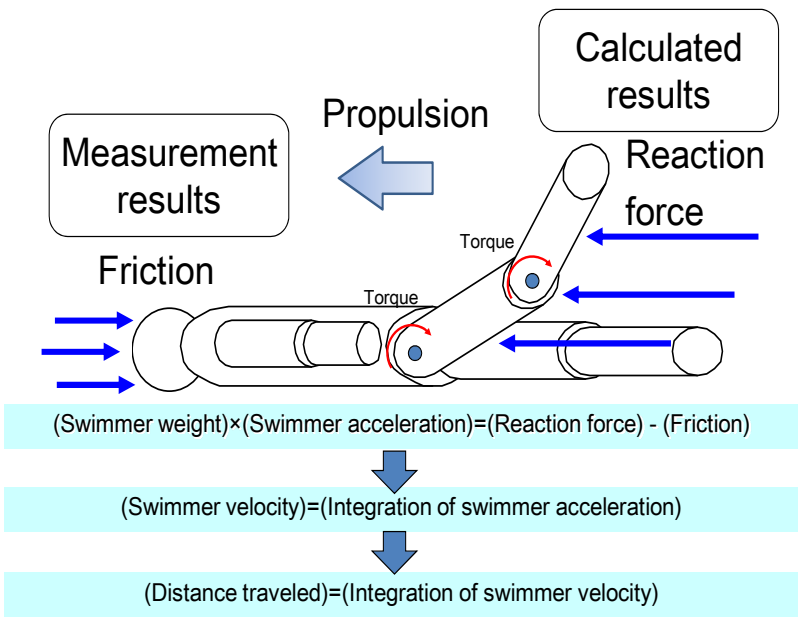

Figure 4. Propulsion of swimmer.
Process 8: The velocity $\boldsymbol{v}_{0}$ is calculated using Eq.12.

Process 9: The distance traveled $x$ is calculated using Eq.13.

In a swimming race, the goal is to swim fast to a given point. Therefore, process 9 must be optimized to increase the distance traveled in time period $t$.

Only the torques of the joints can be controlled. Because of the complex relationship among processes 1-9, it is difficult to obtain the optimal motion. The distance traveled is determined by calculating the propulsion force in process 6 . To simplify the optimization process, the propulsion force is maximized based on the trajectory of the manipulator.

\subsection{Torque Loaded on Joint}

In process 1 , the torques at each joint are defined as a function of time from the start time $0 \mathrm{~s}$ to the end time $t \mathrm{~s}$. The time history of the torque is searched to determine the maximum or minimum propulsion force. By determining a specified input $\tau(t)$, we can calculate the propulsion force in process 6 . In this study, the cost function is defined as the fluid drag on the manipulator surface. The torques $\tau(t)$ are found by maximizing (minimizing) the cost function.

\subsection{State Equation}

In processes $2-5$, the motion of the swimmer is determined from the time history of the torques. The arm and leg motions modeled by the two-link manipulator are calculated by the following state equation:

$$
\boldsymbol{F}=\boldsymbol{M}(\boldsymbol{\theta}) \ddot{\boldsymbol{\theta}}+\boldsymbol{C}(\boldsymbol{\theta}, \dot{\boldsymbol{\theta}})+\boldsymbol{D}(\boldsymbol{\theta}, \dot{\boldsymbol{\theta}})+\boldsymbol{g}(\boldsymbol{\theta})-\boldsymbol{\tau}=0
$$

where $\boldsymbol{M}, \boldsymbol{C}, \boldsymbol{D}$, and $\boldsymbol{g}$ represent the inertial force, centrifugal-coriolis forces, drag, gravity, and buoyancy, respectively. This equation is derived from Eq.5.

\subsection{Cost Function}

To match the model to actual backstroke motion, the trajectory of the manipulators modeling the legs and arms must be artificially controlled. In this study, the optimal motion of the swimmer corresponds to the maximum propulsion force in the direction of movement. The cost function is defined as follows:

$$
\begin{gathered}
J=d_{1}+d_{2}+\gamma_{1}\left(\theta_{i}-a\right)^{2}+\gamma_{2}\left(\theta_{m}-b\right)^{2} \\
(i, m)=(1,2),(3,4),(5,6),(7,8) \\
L=J+\int_{0}^{t} \lambda \cdot \boldsymbol{F} d t
\end{gathered}
$$

where $a, b$, and $\gamma$ represent the objective angles and the arbitrary positive constant. Here $(i, m)=(1,2)$ represents the right arm, $(i, m)=(3,4)$ the left arm, $(i, m)=(5,6)$ the right leg and $(i, m)=(7,8)$ the left leg.

The objective angle makes an artificial backstroke motion. The angle has a specified range so as to match the 
backstroke motion. Thus, constraints are defined. Because of human physical limits, the torque $\tau_{i}$ has a maximum value and is limited as follows:

$$
-150(N \cdot m) \leq \tau_{i} \leq 150(N \cdot m)
$$

The constraint on the angle of joint $\left(A_{2}, A_{6}\right)$ is:

$$
-90^{\circ} \leq \theta_{i} \leq 90^{\circ} \quad i=2,6
$$

The constraint on the angle of joint $\left(A_{3}, A_{7}\right)$ is:

$$
-45^{\circ} \leq \theta_{i} \leq 45^{\circ} \quad i=3,7
$$

The constraint on the angle of joint $\left(A_{4}, A_{8}\right)$ is:

$$
0^{\circ} \leq \theta_{i} \leq 45^{\circ} \quad i=4,8
$$

\subsection{Numerical Algorithm to Find Optimal Trajectory}

The algorithm is as follows: (Figure 5)

Step 1: Set the time history of the torque of joint $A_{i}$ to $\tau_{i}=0$.

Step 2: Calculate the state equation from start time $0 \mathrm{~s}$ to end time $t \mathrm{~s}$ using the Runge-Kutta method. Obtain the parameters $\theta, \dot{\theta}, \ddot{\theta}$ at every time step and store them in memory. The parameters $\dot{\theta}, \ddot{\theta}$ represent the angular velocity and the angular acceleration.

Step 3: Modify the angle to satisfy Eqs.18-20.

Step 4: Use the parameters $\theta, \dot{\theta}, \ddot{\theta}$ from step 2 to calculate the adjoint equations (Eqs.21-22) from the end time to the start time using the end-time condition given in Eq.23. Solve these equations by the Runge-Kutta method, and store the adjoint variables at every time step.

$$
\begin{gathered}
\frac{\partial L}{\partial \theta_{i}}-\frac{d}{d t}\left(\frac{\partial L}{\partial \dot{\theta}_{i}}\right)=0 \quad i=1,2 \\
\frac{\partial L}{\partial \dot{\theta}_{i}}-\frac{d}{d t}\left(\frac{\partial L}{\partial \ddot{\theta}_{i}}\right)=0 \quad i=1,2 \\
\frac{\partial L(t)}{\partial \theta_{i}}=0, \quad \frac{\partial L(t)}{\partial \dot{\theta}_{i}}=0, \quad \frac{\partial L(t)}{\partial \ddot{\theta}_{i}}=0 \quad i=1,2
\end{gathered}
$$

where the parameter $t$ represents the end time.

Step 5: Obtain the gradient of the Lagrange function using the state variables and adjoint variables:

$$
\frac{\partial L}{\partial \tau}=\int_{0}^{t} \lambda \cdot \frac{\partial \mathbf{F}}{\partial \tau} d t=-\int_{0}^{t} G d t
$$

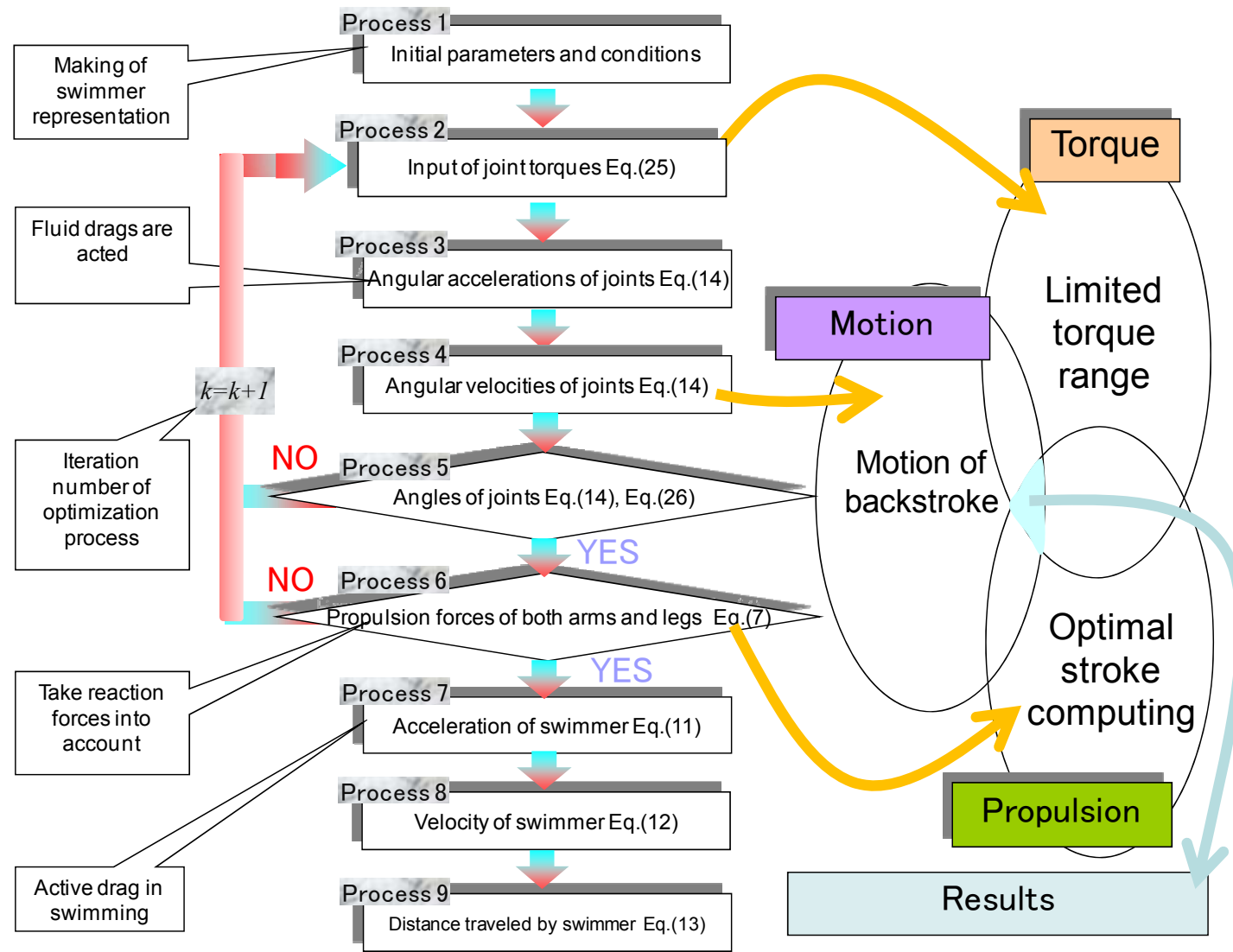

Figure 5. Algorithm. 
Step 6: If the gradient of the Lagrange function is approximately zero, the Lagrange function has reached an extreme value. Obtain the optimal motion using the time history of the torques. If the Lagrange function has not reached an extreme value, continue to step 7 .

Step 7: Update the time history of the torques using the gradient method:

$$
\tau_{i,(k+1)}=\tau_{i,(k)}+\alpha G_{i,(k)} \quad(i=1,2)
$$

where $\alpha$ represents a small value. The index $k$ represents the iteration number of the optimization process as shown in Figure 5.

Step 8: If $a$ and $b$ are sufficiently close to the objective angles within time $t$ as shown in Eq.26, the optimal motion of the swimmer has been obtained. Otherwise, continue to step 9 .

$$
\gamma_{1}\left\{\theta_{1}(t)-a\right\}^{2}+\gamma_{2}\left\{\theta_{2}(t)-b\right\}^{2} \cong 0
$$

Step 9: Increase the current time $t$ by $t / 1000$. Return to step 2 .

\section{RESULTS}

Backstroke is simulated using the algorithm presented in Section 4. The specifications are summarized in Table 1. The gravity $g$, water density $\rho$, and drag coefficient $C_{i}$ are set to be $9.8 \mathrm{~m} / \mathrm{s}^{2}, 1000 \mathrm{~kg} / \mathrm{m}^{3}$, and 1.0 , respectively.

The initial position $(t=0.0 \mathrm{~s})$ of the swimmer is defined as shown in Figure 2. The initial angles are set to be $0.0^{\circ}$. The time history of the torques is input for the right arm (joints $A_{1}$ and $A_{2}$ ) and the right leg (joints $A_{3}$ and $A_{4}$ ), as shown in Figures $\mathbf{7}$ and $\mathbf{8}$. The time history of the torque that is $0.15 \mathrm{~s}$ late is input for the left arm $\left(A_{5}, A_{6}\right)$ and the left leg $\left(A_{7}, A_{8}\right)$.

Figure 6 shows a side view and an oblique view of the optimal motion of the swimmer. The figure indicates that the model provides a good approximation of backstroke motion, although the motion is restricted to two dimensions.

The time history of the torques for joints $A_{1}$ and $A_{2}$ is shown in Figure 7. At about $0.36 \mathrm{~s}$, torque $\tau_{1}$ rapidly changes from a negative rotation to a positive rotation.

Table 1. Specifications of swimmer representation.

\begin{tabular}{ccccc}
\hline & $\begin{array}{c}\text { Mass } \\
(\mathrm{kg})\end{array}$ & $\begin{array}{c}\text { Length } \\
(\mathrm{m})\end{array}$ & $\begin{array}{c}\text { Radius } \\
(\mathrm{m})\end{array}$ & Form \\
\hline Head & 5.0 & 0.2 & 0.1 & Sphere \\
Body & 35.0 & 0.7 & 0.1 & Cylinder \\
Upper arm & 5.0 & 0.4 & 0.07 & Cylinder \\
Forearm & 5.0 & 0.4 & 0.06 & Cylinder \\
Thigh & 5.0 & 0.4 & 0.07 & Cylinder \\
Lower thigh & 5.0 & 0.4 & 0.06 & Cylinder \\
\hline$\left(M=60 \mathrm{~kg}, H_{s}=1.7 \mathrm{~m}\right)$ & & & &
\end{tabular}

$\left(M=60 \mathrm{~kg}, H_{s}=1.7 \mathrm{~m}\right)$
On the other hand, torque $\tau_{2}$ changes from a positive rotation to a negative rotation. The maximum propulsion force occurs with the rapid snap of one arm. After that, torque $\tau_{2}$ changes to a positive rotation again because the angles are restricted to the range given in Eq.18.

Figure 8 shows the time history of the torque for the right leg. Optimization can only be performed in the time span from 0.5 to $0.6 \mathrm{~s}$ because the maximum propulsion force occurs in this time span.

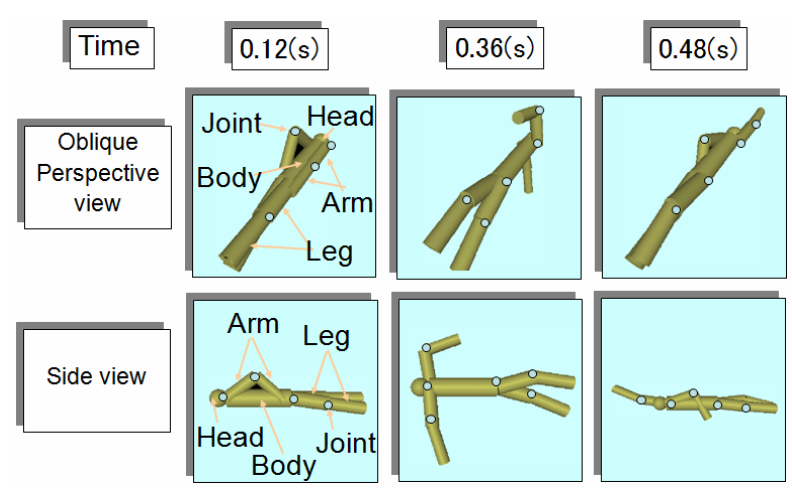

Figure 6. Backstroke motion.

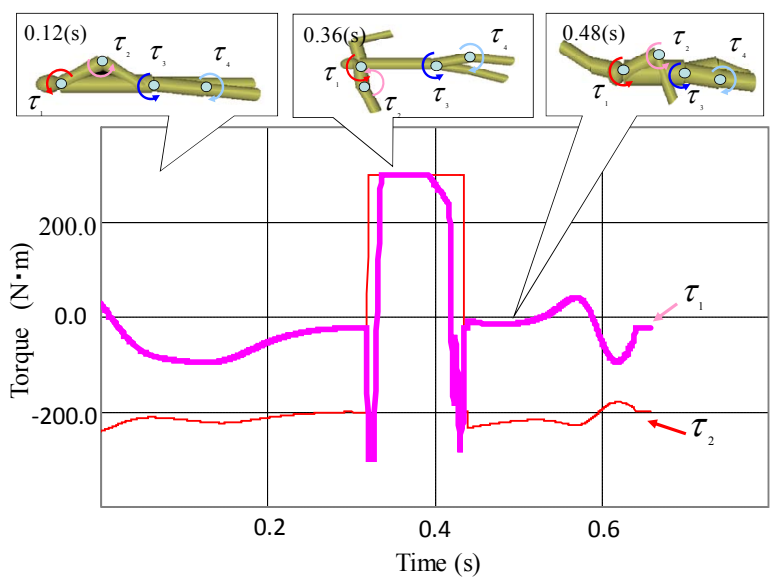

Figure 7. Time history of joint torques for right arm shown in Figure 2.

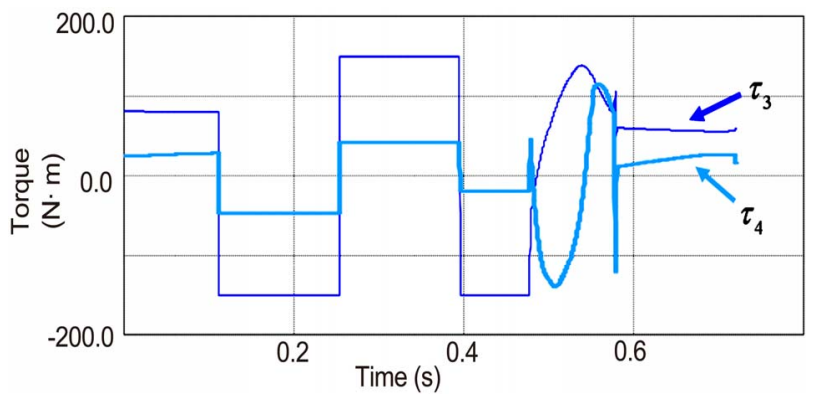

Figure 8. Time history of joint torques for right leg shown in Figure 2. 
Figure 9 shows the time histories of the propulsion force for one arm and one leg. The propulsion force of the leg is smaller than that of the arm. In these time histories, mountains and valleys alternate. The literature indicates that the propulsion force of an actual swimmer depends on the force exerted by the arm [11]. In the literature [10], the total drag force of a woman under water is calculated by CFD (computational fluid dynamics). The swimmer proceeds by the propulsion equivalent to this total drag force (about $150 \mathrm{~N}$ ), which is generated when the swimmer proceeds with a velocity of about 1.5 $\mathrm{m} / \mathrm{s}$.

In this study, the propulsion force (about $0(\mathrm{~N})$ 600(N), as shown in Figure 9) is much larger than this force reported in the literature [11]. In this study, the drag coefficient is assumed to be 1.0; in the literature [10], it is about 0.28-0.4. The $k-\varepsilon$ turbulent model is applied to the computational model developed in this study. CFD analysis enables to calculate the turbulent flow in a local area. Therefore, as compared to the drag coefficient employed in this study, the drag coefficient used in the literature [10] may better capture the actual fluid phenomenon. However, this approach of using an FEM (finite element method) mesh cannot be used to simulate the motion of a swimmer. This is because we need to deform the FEM mesh according to the swimmer's motion. As the elements of the FEM mesh become irregular due to the deformation, the deformation partly causes a numerical vibration in the fluid analysis and leads to negative volumes of mesh elements. Therefore, it may be difficult to simulate the motion of a real swimmer by

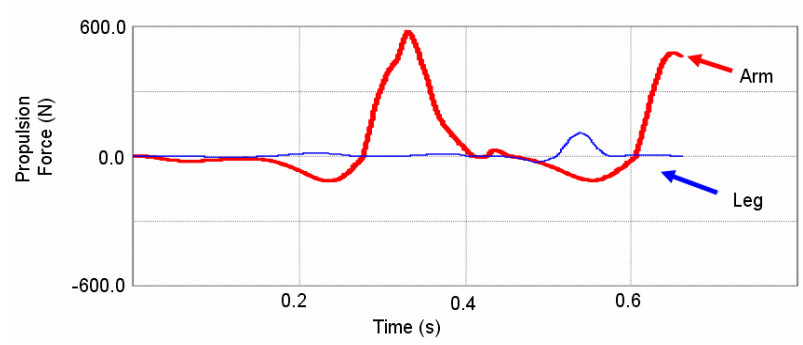

Figure 9. Time histories of propulsion force for one arm and one leg.

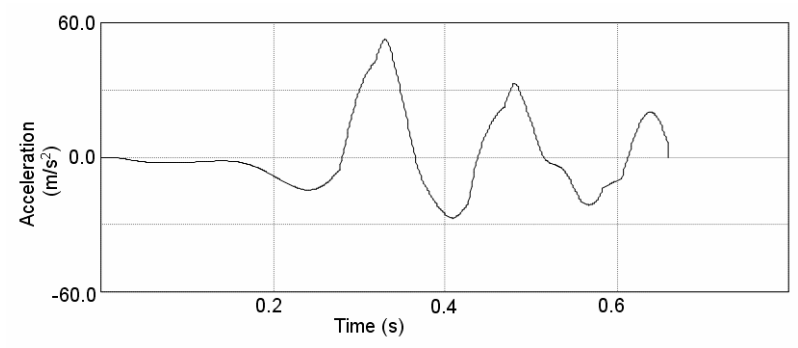

Figure 10. Time history of acceleration. using CFD techniques based on the FEM (or finite volume method, etc.).

Figure 10 shows the time history of the acceleration; there are three positive peaks. During these peaks, the maximum propulsion forces arise from arm strokes.

The velocity is shown in Figure 11. It becomes negative in the time span from 0.0 to $0.3 s$; this is because in this model, no boundary condition is set for the interface between water and air. Therefore, a negative propulsion force occurs because of the negative acceleration. The velocity range $(0.0-1.5 \mathrm{~m} / \mathrm{s})$ agrees quite closely with that of an actual swimmer [21]. In the literature [9,22], backstroke analysis performed using the SWUM model is presented. The mass and the body length in this SWUM model are $64.9 \mathrm{~kg}$ and $1.705 \mathrm{~m}$, respectively, and the drag coefficient for the normal direction is set to be 1.08. With respect to these parameters, this SWUM model almost agrees with that used herein. The maximum velocity calculated using the SWUM model instantaneously attains a value of about $1.5 \mathrm{~m} / \mathrm{s}$. On the other hand, the maximum velocity calculated by the model used in this study instantaneously attains a value of about $1.6 \mathrm{~m} / \mathrm{s}$. Therefore, the model used in this study is slightly superior to the SWUM model.

The time history of the distance traveled is shown in Figure 12. Because of the negative velocity in the time span from 0.0 to $0.3 \mathrm{~s}$, the distance becomes negative. After $0.3 s$, the swimmer consistently moves in the positive direction because of the positive velocity.

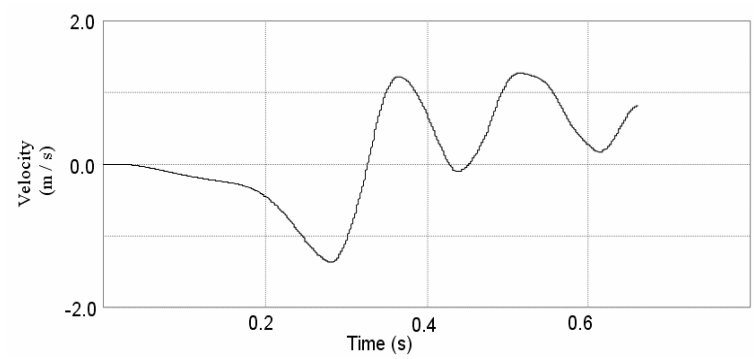

Figure 11. Time history of velocity.

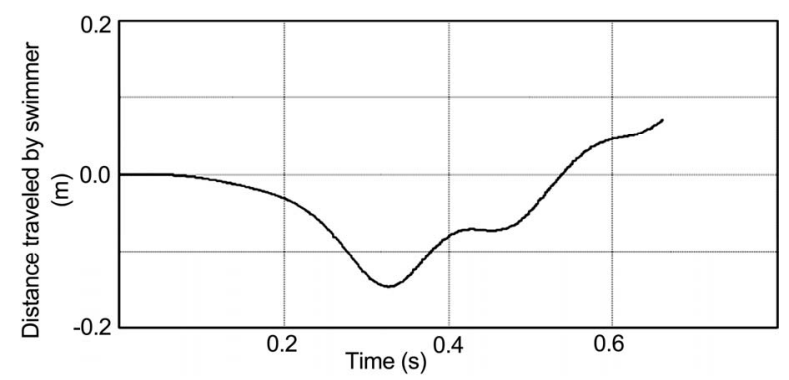

Figure 12. Time history of distance traveled. 


\section{CONCLUSIONS}

We developed a model by using the dynamics of an underwater manipulator. An algorithm was constructed on the basis of the manipulator dynamics. The results obtained by this algorithm qualitatively agreed with the experimental results.

In this study, a swimmer model providing the optimal motion is presented. The optimization method mainly consists of the probabilistic approach (a genetic algorithm, simulated annealing, etc.) and the deterministic approach (adjoint variable method, etc.). In case the motion is restricted to be $2 \mathrm{D}$, the optimizer can easily search for the optimal value. If the $2 \mathrm{D}$ motion is extended to $3 \mathrm{D}$ motion in this study model, it may be difficult to search for the optimal value. By using the deterministic approach (the adjoint variable method), it is highly possible to ensure that the optimizer searches for the local minimum by increasing the number of parameters. The deterministic approach also demands the stationary condition based on the variational method. The adjoint equation derived by the stationary condition has strong nonlinear characteristics. This nonlinearity causes numerical instability. In the future study, the 3D motion of a swimmer will be simulated by using the probabilistic approach, which does not need mathematical formulation.

\section{REFERENCES}

[1] Laffite, L., Vilas-Boas, J.P., Demarle, A., Silva, J., Fernandes, R. and Billat, V. (2004) Changes in physiological and stroke parameters during a maximal 400-m free swimming test in elite swimmers. Canadian Journal of Applied Physiology, 229, S17-31.

[2] Silva, A.J., Machado, R. V., Guidetti, L., Bessone, A. F., Mota, P., Freitas, J. and Baldari, C. (2007) Effect of creatine on swimming velocity, body composition and hydrodynamic variables. Journal of Sports Medicine and Physical Fitness, 47(1), 58-64.

[3] Soons, B., Colman, V., Persyn, U. and Silva, A. (2003) Specific movement variables important for performance in different breaststroke styles. In Biomechanics and Medicine in Swimming, IX, 295-300.

[4] Barbosa, T.M., Bragada, J.A., Reis, V.M., Marinho, D.A., Carvalho, C. and Silva, A.J. (2010) Energetics and biomechanics as determining factors of swimming performance: updating the state of the art. Journal of Science and Medicine in Sport, 13(2), 262-269.

[5] Silva, A., Costa, A.M., Oliveira, P.M., Reis V., Saavedra, J., Perl, J., Rouboa, A. and Marinho, D. (2007) The use of neural network technology to model swimming performance. Journal of Sports Science and Medicine 6(1), 117-125.

[6] Arellano, R., Nicoli-Terrés, J.M. and Redondo, J.M. (2006) Fundamental hydrodynamics of swimming propulsion. Portuguese Journal of Sport Sciences, 6(Suppl. 2), $15-20$.

[7] Härtel, T. and Axel, S. (2008) Evaluation of start tech- niques in sports swimming by dynamics simulation. The Engineering of Sport, 7(1), 89-96.

[8] Kwatra, N., Wojtan, C., Carlson, M., Essa, I., Mucha, P.J., and Turk, G. (2010) Fluid simulation with articulated bodies. IEEE Transactions on Visualization and Computer Graphics, 16(1), 70-80.

[9] Nakashima, M., Satou, K. and Mura, Y. (2007) Development of swimming human simulation model considering rigid body dynamics and unsteady fluid force for whole body. Journal of Fluid Science and Technology, 2(1), 56-67.

[10] Zaïdi, H., Taïar, R., Fohanno, S. and Polidori, G. (2008) Analysis of the effect of swimmer's head position on swimming performance using computational fluid dynamics. Journal of Biomechanics, 41(6), 1350-1358.

[11] Lecrivain, G., Slaouti, A., Payton, C. and Kennedy, I. (2008) Using reverse engineering and computational fluid dynamics to investigate a lower arm amputee swimmer's performance. Journal of Biomechanics, 41(13), 2855-2859.

[12] Silva, A.J., Rouboa, A., Moreira, A., Reis, V.M., Alves, F., Vilas-Boas, J.P. and Marinho, D.A. (2008) Analysis of drafting effects in swimming using computational fluid dynamics. Journal of Sports Science and Medicine, 7(1), 60-66.

[13] Marinho, D.A., Reis, V.M., Alves, F.B., Vilas-Boas, J.P., Machado, L., Silva, A.J. and Rouboa, A.I. (2009) Hydrodynamic drag during gliding in swimming. Journal of Applied Biomechanics, 25(3), 253-257.

[14] Cohen, R.C.Z., Cleary, P.W. and Mason, B. (2009) Simulations of human swimming using smoothed particle hydrodynamics. 7th International Conference on CFD in the Minerals and Process Industries, Commonwealth Scientific and Industrial Research Organisation.

[15] Marinho, D., Barbosa, T., Reis, V.M., Kjendlie, P.-L. and Alves, F.B., (2010) Swimming propulsion forces are enhanced by a small finger spread. Journal of Applied Biomechanics, 26(1), 87-92.

[16] Marinho, D.A., Barbosa, T.M., Kjendlie, P.L., VilasBoas, J.P., Alves, F.B., Rouboa, A.I. and Silva, A.J. (2009) Swimming simulation: A new tool for swimming research and practical applications. Computational Fluid Dynamics for Sport Simulation, 33-61.

[17] Rouboa, A., Silva, A., Leal, L., Rocha, J. and Alves, F. (2006) The effect of swimmer's hand/forearm acceleration on propulsive forces generation using Computational Fluid Dynamics. Journal of Biomechanics, 39(7), 12391248.

[18] Shinohara, K., Furukawa, T. and Yagawa, G. (2002) Simulation and sub-optimal motion planning of a swimmer under hydrodynamics. Transactions of the Japan Society of Mechanical Engineers, 68(673), 2643-2650.

[19] Takagi, H., Shimizu, Y. and Kodan, N. (1999) A hydrodynamic study of active drag in swimming. JSME International Journal Series B, 42(2), 171-177.

[20] Marinho, D.A., Barbosa, T.M., Kjendlie, P.L., Mantripragada, N., Vilas-Boas, J.P., Machado, L., Alves, F.B., Rouboa, A.I. and Silva, A.J. (2010) Modelling hydrodynamic drag in swimming using computational fluid dynamics. Computational Fluid Dynamics, 17, 391-404.

[21] Arellano, R., Pardillo, S. and Gavilan, S. (2002) Underwater undulatory swimming: Kinematic characteristics, 
vortex generation and application during the start, turn and swimming strokes. Proceedings of the 20th International Symposium on Biomechanics in Sports, Universidad de Granada.

[22] Nakashima, M. (2007) Analysis of breast, back and but- terfly strokes by the swimming human simulation model swum. In: Kato, N. and Kamimura, S., Eds., Biomechanisms of Animals in Swimming and Flying-Fluid Dynamics, Biomimetic Robots, and Sports Science, SpringerVerlag, 361-367. 